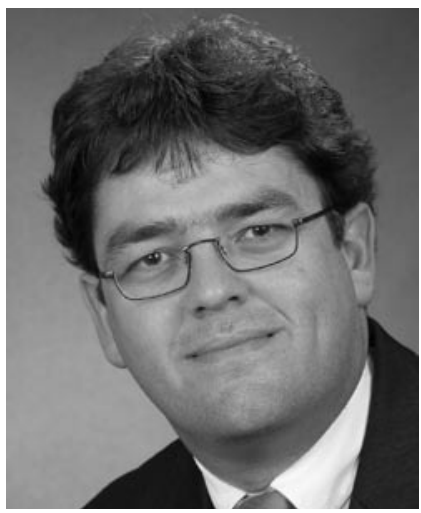

Thomas Hess

\section{Controlling \\ und Datenqualität}

\section{Liebe Leser,}

ein Controlling ohne Unterstützung durch Anwendungssysteme ist schon seit Jahren nicht mehr vorstellbar. In kleinsten Unternehmen reichen dafür häufig einfache Tabellenkalkulationssysteme aus. Data-Warehouse-Systeme und multidimensionale Datenbanken sowie darauf aufbauende Management-Cockpits prägen heute das Bild in größeren Unternehmen. Konzeption, Einführung, Betrieb und Weiterentwicklung derartiger Systeme sind häufig (neben den originären Personalkosten) der größte Posten im Budget einer Controlling-Abteilung. Umso wichtiger ist es, dass diese Systeme auch ihren vollen Nutzen entfalten. Dieser lässt sich aber nur erreichen, wenn die Daten in den Systemen korrekt, vollständig und konsistent sind - keinesfalls eine Selbstverständlichkeit. Die Folgen mangelhafter Datenqualität liegen auf der Hand: falsche Entscheidungen werden getroffen, Kunden erhalten fehlerhafte Mitteilungen, Lieferanten werden falsch informiert etc.

Mit dem Thema Datenqualität wird die Schnittstelle zwischen Controlling und IT nochmals ausgebaut. Ging es an dieser Schnittstelle lange Zeit "nur" um Konzeption, Entwicklung, Betrieb und Weiterentwicklung der "richtigen Systeme" zur Unterstützung des Controllings, so kommt nun auch noch die Sicherstellung der Datenqualität in den geschaffenen Systemen hinzu. Natürlich hat diese Aufgabe auch technische Aspekte, etwa wenn es um die technisch fehlerhafte Übertragung von Daten z. B. zwischen zwei Systemen geht. Ohne die Veränderung operativer Prozesse oder die Vereinheitlichung von Kennzahlendefinitionen lässt sich das Thema aber häufig nicht in den Griff bekommen. Genauso lassen sich die Kosten fehlerhafter Datenbestände, auf deren Basis über größere Datenqualitätsmanagement-Projekte entschieden wird, alleine aus der technischen Perspektive nicht beziffern. Jeder Controller sollte daher auch zum Qualitätsmanager der eigenen Daten werden - die IT-Abteilung kann dabei nur begrenzt helfen.

In vorliegendem Schwerpunktheft wollen wir Ihnen daher aktuelle Ansätze zum Management der Datenqualität im Controlling vorstellen und gleichzeitig aufzeigen, wie sich diese in der Controlling-Praxis umsetzen lassen. Boris Otto und Henning Hinderer haben auf Basis vierer Fallbeispiele einen Architekturentwurf für das Datenqualitätsmanagement im Bereich des Lieferantencontrollings erstellt. Die Fallbeispiele liefern gleichzeitig praktische Handlungsempfehlungen für die Übertragung des Modells auf das eigene Unternehmen. Jan Philipp Rohweder berichtet über ein eingängiges Konzept für das Controlling der Datenqualität, die „Netto-Datenbank-Qualität", und zeigt auf, wie die dadurch gewonnene Transparenz zur erfolgreichen Steuerung des Marketing und CRM bei der Roche Diagnostics GmbH genutzt werden konnte. Bernd Heinrich und Mathias Klier stellen in ihrem Beitrag zwei wissenschaftlich fundierte und gleichzeitig praxisnahe Metriken zur Messung der Datenqualität vor. Die Konstruktion und Anwendung der Datenqualitätsmetriken werden von den Autoren im Bereich des Kundenwertcontrollings eines Finanzdienstleisters illustriert

ZurVertiefung geben Ihnen Ulrike Baumöl und Martina Meschke einen Überblick über die internationale Forschung an der Schnittstelle zwischen Controlling und Datenqualität. Daneben haben wir Ihnen in der Rubrik IT-Lösungen verschiedene Ansatzmöglichkeiten zur IT-Unterstützung des Datenqualitätsmanagements zusammengestellt. Falls Sie sich weiterführend informieren wollen, finden Sie außerdem eine Liste mit lesenswerter Literatur zum Heftthema.

Wir hoffen, dass Sie aus diesen (und natürlich auch den weiteren) Beiträgen wertvolle Anregungen für die Gestaltung Ihrer eigenen Arbeit erhalten.

\section{Mit herzlichen Grüßen}

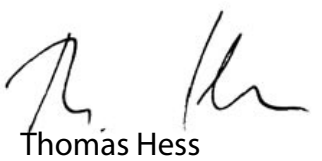

